\title{
Brugada Syndrome with Aborted Sudden Cardiac Death Related to Liquorice-Induced Hypokalemia
}

\author{
Hikmet Yorgun ${ }^{a}$ Hakan Aksoy ${ }^{a}$ Mehmet Ali Şendur ${ }^{b} \quad$ Ahmet Hakan Ateş ${ }^{a}$ \\ Ergün Barış Kaya ${ }^{a}$ Kudret Aytemir ${ }^{a} \quad$ Ali Oto $^{a}$
}

Departments of a Cardiology and ${ }^{b}$ Internal Medicine, Hacettepe University, Ankara, Turkey

\section{Key Words}

Brugada syndrome • Cardiac arrest • Liquorice •

Hypokalemia

\begin{abstract}
Objective: It was the aim of this study to report an aborted cardiac arrest due to ventricular fibrillation and electrocardiographic changes consistent with Brugada syndrome due to liquorice-induced hypokalemia. Clinical Presentation and Intervention: Ventricular fibrillation was witnessed in a 50-year-old woman who was admitted to our emergency department with a history of liquorice ingestion, a herbal product. After stopping liquorice ingestion, the Brugada-like electrocardiographic pattern changed progressively with potassium replacement. A diagnosis of Brugada syndrome was made after the ajmaline challenge test. The patient was discharged with an implantable cardioverter defibrillator and had an uneventfull follow-up. Conclusion: This report illustrates the importance of the investigation for herbal medications in the detailed history of a patient in the cases of electrolyte disturbances and the potential role of hypokalemia in the induction of malignant arrhythmia in Brugada syndrome.

Copyright $\odot 2010$ S. Karger AG, Basel
\end{abstract}

\section{Introduction}

Brugada syndrome is characterized with electrocardiographic (ECG) findings of apparent or real right bundle branch block and ST segment elevation in the right precordial leads in the absence of long QT intervals and any structural heart disease. This syndrome is associated with an increased risk of sudden cardiac death due to malignant ventricular dysrhythmias [1]. Several clinical conditions and electrolyte imbalances, including hypokalemia, can provoke ECG changes and ventricular dysrhythmias in Brugada syndrome [2]. We present a case report of Brugada syndrome diagnosed after aborted sudden cardiac death due to hypokalemia caused by liquorice ingestion, a herbal product.

\section{Case Report}

A 50-year-old woman admitted to our emergency department with complaints of generalized weakness, chest pain and sweating for $4 \mathrm{~h}$. Relatives of the patient stated that she had a syncopal attack $1 \mathrm{~h}$ before complaints of palpitation and dizziness, which she had never described before. She did not have any cardiovascular or systemic illness and was not taking any medication. She had no prior personal history of syncope or aborted cardiac arrest and her family history was also negative for sudden cardiac death. On admission, her pulse rate was regular with 64 beats $/ \mathrm{min}$, blood pressure was $170 / 100 \mathrm{~mm} \mathrm{Hg}$ and her body temperature was $37.1^{\circ} \mathrm{C}$. She was confused, and physical examination was unremarkable

Hikmet Yorgun, MD

Department of Cardiology

Hacettepe University Faculty of Medicine

TR-06100 Sihhiye, Ankara (Turkey)

Tel. +90 312305 1780, Fax +90 312305 4137, E-Mail yorgunhikmet@yahoo.com
(C) 2010 S. Karger AG, Basel

www.karger.com 


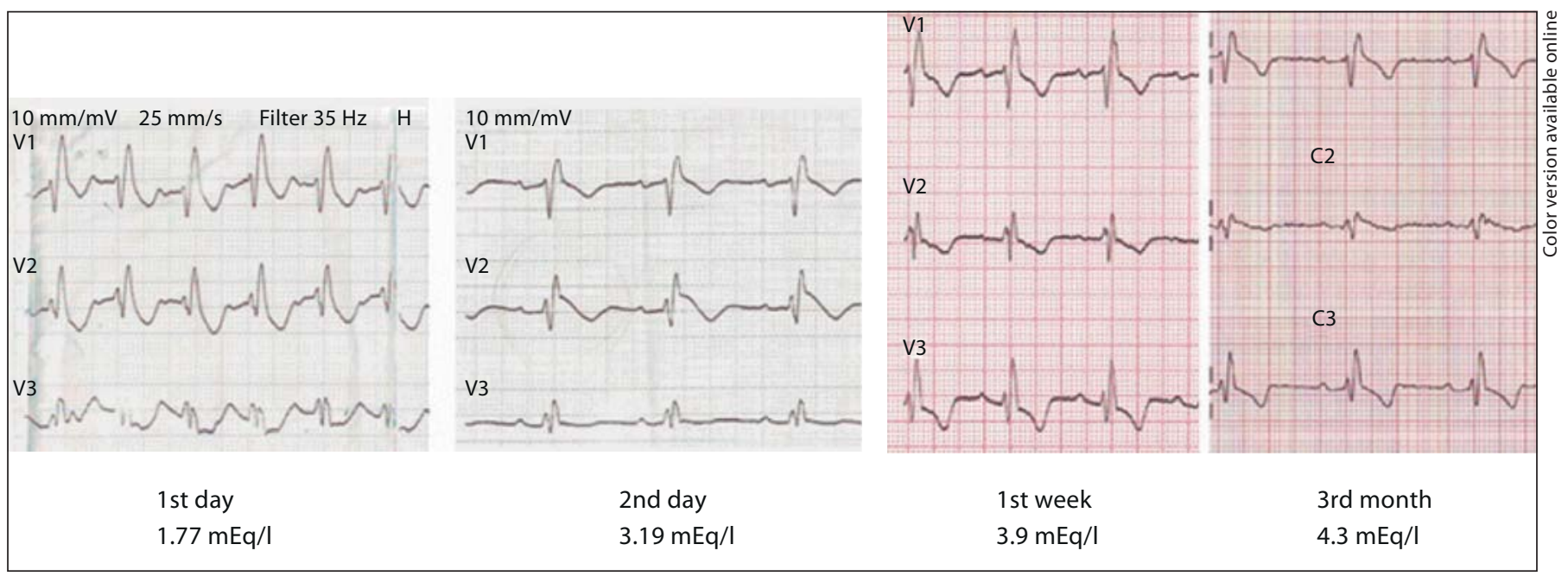

Fig. 1. ECG changes from admission to the 3rd month after the potassium replacement seen in V1-V3.

except for a mild apical systolic murmur. ECG showed sinus rhythm, a ventricular rate of 112 beats/min and right bundle branch block. The corrected QT interval was $0.45 \mathrm{~s}$, with a J point elevation of $2 \mathrm{~mm}$ and a PR interval of $0.22 \mathrm{~s}$. Cranial computed tomography was taken to rule out a cerebrovascular accident, which revealed normal findings. Complete blood count and cardiac troponins were in normal limits, but blood biochemistry disclosed hypokalemia $(1.77 \mathrm{mEq} / \mathrm{l})$ and a mild disturbance in liver function tests (ALT $84.5 \mathrm{U} / \mathrm{l}$, AST $102 \mathrm{U} / \mathrm{l}$, GGT 78.8 U/l). The patient had neither a history of vomiting and diarrhea nor recent medication usage that might cause the electrolyte disturbance, except for a herbal product that contained liquorice. Detailed history revealed that she had been drinking 2 cups of liquorice extract a day for 3 months to relieve generalized weakness. During monitoring in the emergency department, ventricular fibrillation was witnessed and was successfully converted to sinus rhythm with a single shock of $300 \mathrm{~J}$. Intravenous potassium replacement was started with $20 \mathrm{mEq} / \mathrm{h}$ and the control level was $2.65 \mathrm{mEq} / \mathrm{l}$ after 4 h. A 12-lead ECG taken $24 \mathrm{~h}$ after admission showed sinus rhythm with a coved-type ST segment elevation on precordial leads V1-V2 followed by a deep T-wave inversion; the serum potassium level at the time was $3.19 \mathrm{mEq} / \mathrm{l}$ (fig. 1). The corrected QT interval was $0.44 \mathrm{~s}$, with a J point elevation of $1 \mathrm{~mm}$ in $\mathrm{V} 1$ and 2.5 $\mathrm{mm}$ in V2 and a PR interval of $0.22 \mathrm{~s}$. Echocardiography showed mild mitral and tricuspid insufficiency with normal systolic function and right ventricular dimensions. Coronary angiography revealed normal coronary vessels. At 1 week, plasma $\mathrm{K}^{+}$increased to $3.9 \mathrm{mEq} / \mathrm{l}$ and QRS further narrowed. With the recovery from hypokalemia, no dysrhythmia was recorded. Oral potassium replacement was given for 1 week and, after normal serum levels were reached, the patient was discharged from hospital uneventfully. After 3 months, the serum potassium level was $4.3 \mathrm{mEq} / 1$ after withholding liquorice ingestion without further potassium supplements. The corrected QT interval was $0.44 \mathrm{~s}$, J point elevation was $1 \mathrm{~mm}$ in $\mathrm{V} 1$ and $2 \mathrm{~mm}$ in $\mathrm{V} 2$, and the PR interval was $0.22 \mathrm{~s}$ (fig. 2). Because the Brugada phenotype had appeared with accompanying hypokalemia, an intravenous ajmaline challenge was performed 3 months later. Ajmaline at a dose of $1 \mathrm{mg} / \mathrm{kg}$ was administered over $5 \mathrm{~min}$ in the electrophysiology laboratory. The ajmaline challenge revealed a coved-type ST segment elevation in V1-V2 as well as a ventricular rate after 14 min (fig. 3). These results confirmed the diagnosis of Brugada syndrome and an implantable cardioverter defibrillator was implanted for secondary prevention of sudden cardiac death.

\section{Discussion}

A Brugada-like ECG pattern can be seen in several conditions other than Brugada syndrome like antiarrhythmic agents - such as flecainide, procainamide or ajmaline - physical stress, hypothermia and electrolyte disturbances like hypokalemia and hyperkalemia [2-4]. The present case highlights the effects of hypokalemia on the ECG of a patient diagnosed with Brugada syndrome. Brugada syndrome is characterized by ECG findings of apparent or real right bundle branch block and ST segment elevation in the right precordial leads with a high risk of ventricular tachycardia/ventricular fibrillation in the absence of structural heart disease, as described by Brugada and Brugada [5]. ECG findings may change over time and even normalization in ECG may be observed. The pathophysiologic mechanism underlying these ECG changes is an outward shift in the ionic current balance during the early phase 1 of the action potential causing a marked accentuation of the action potential notch, predominantly in the right ventricular epicardium [6].

Glycyrrhizic acid (GA) is the active molecule of liquorice which is converted to glucuronic acid and glycyr- 


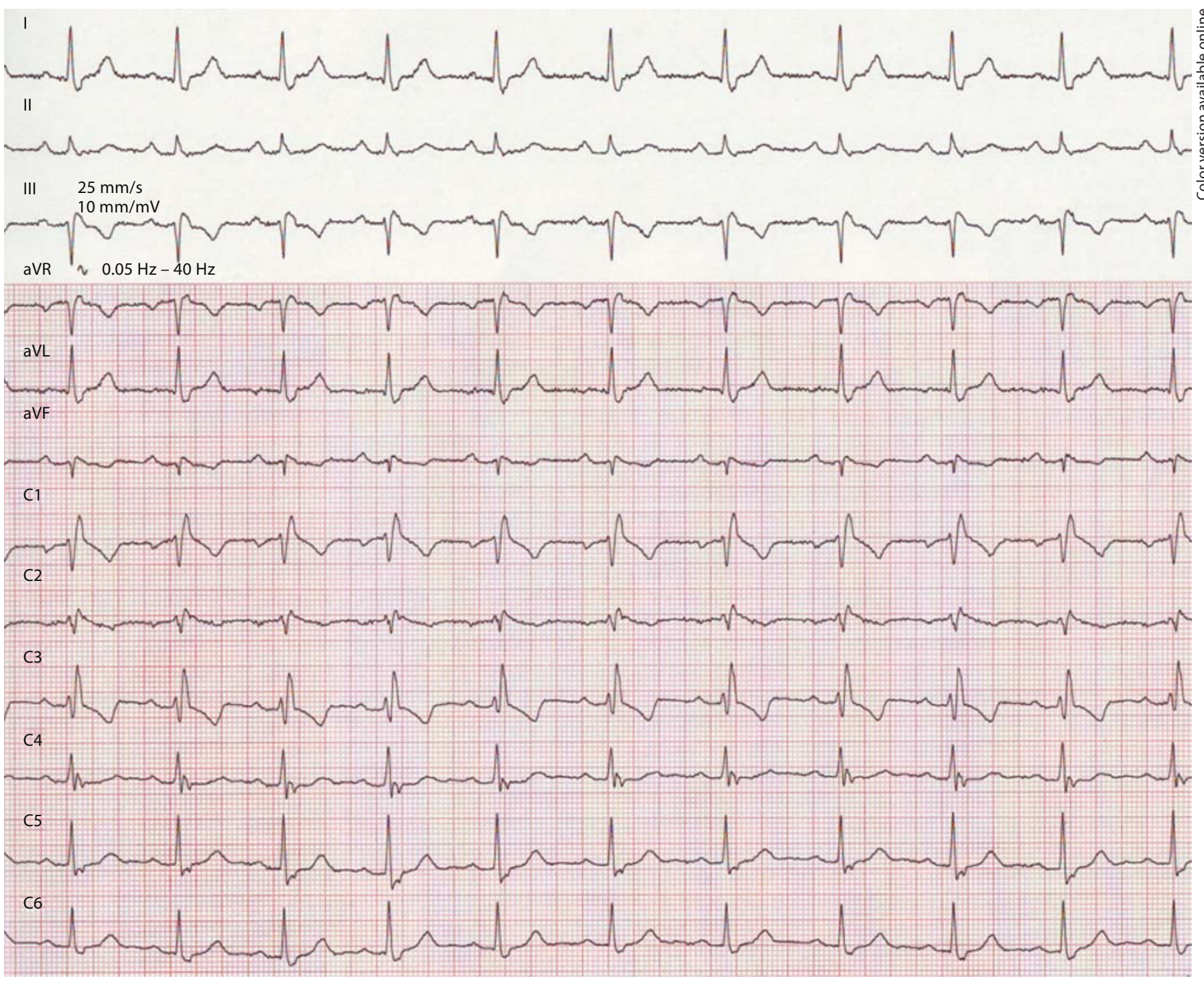

Fig. 2. Baseline ECG before ajmaline infusion.

rhetinic acid in the intestine, and plays a role in the inhibition of the enzyme 11- $\beta$-hydroxysteroid dehydrogenase type 2 . As this enzyme catalyses the inactivation of cortisol to cortisone, potassium excretion in the cortical collecting duct is increased due to mineralocorticoid effects of cortisol, which is the result of liquorice ingestion [7]. Sigurjonsdottir et al. [8] stated that liquorice intake of $100 \mathrm{~g} /$ day, which contains $150 \mathrm{mg}$ of GA, causes an increase in systolic blood pressure of $15 \mathrm{~mm} \mathrm{Hg}$ in hypertensive patients and of $3.5 \mathrm{~mm} \mathrm{Hg}$ in normotensive subjects. Although a dose-response relation between GA amount and cortisolcortisone ratio was shown by Krähenbühl et al. [9], there were also case reports [10] describing clinical effects due to low-dose liquorice intake. The mechanism of susceptibility to the effects of GA is not clear, but a mechanism was described regarding the tendency for females, considering the effects of estrogens on mineralocorticoid receptors [11].

In this patient, liquorice-induced hypokalemia was suspected to provoke the ECG changes and ventricular fibrillation. Also, hypertension was thought to be the result of liquorice ingestion as the patient remained normotensive after cessation of liquorice extract. Cardiac arrest due to hypokalemia-induced ventricular fibrillation related to QT prolongation was reported previously [12]. However, in this case, only a small increase in corrected QT interval and PR interval was observed in our patient. 


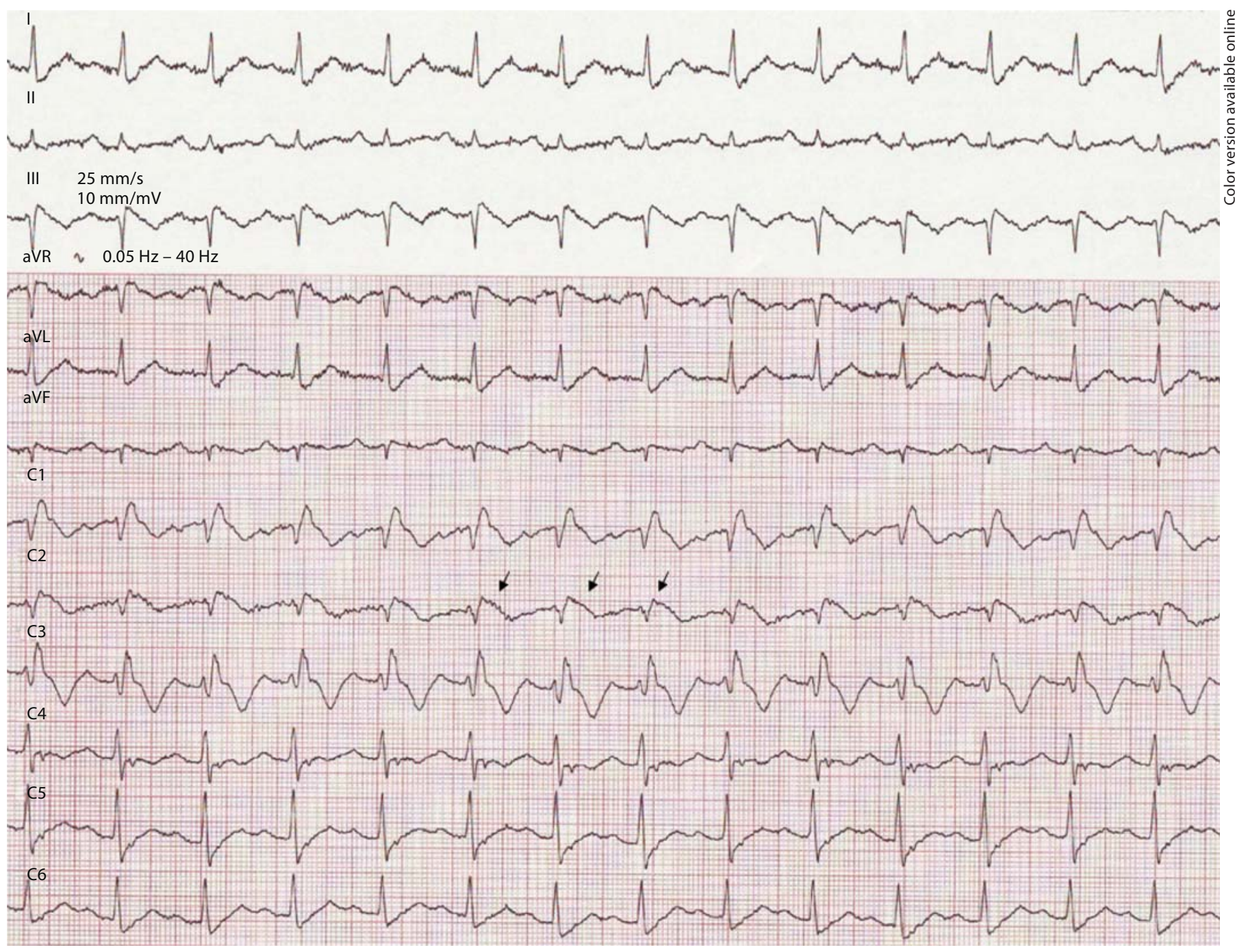

Fig. 3. ECG taken at the end of the ajmaline challenge test showing coved-type ST segment elevation in V1-V2 (arrows).

These findings may be due to a loss of function mutation in SCN5A gene, which is often observed in Brugada syndrome patients manifesting first-degree atrioventricular block, as in our patient. Coved-type ST segment elevation was reduced with potassium replacement suggesting that hypokalemia contributed to the induction of ventricular fibrillation via a Brugada syndrome mechanism, rather than an acquired long QT mechanisms. Araki et al. [2] and Notarstefano et al. [13] reported cases with ventricular dysrhythmias and described the role of hypokalemia as the suggested mechanism in the occurrence of ventricular tachycardia and fibrillation in Brugada syndrome. The ECG changes may vary over time in a dynamic manner in Brugada syndrome, as in our case report.

\section{Conclusion}

In the present case, a rare case of hypokalemia was caused by liquorice ingestion, a herbal product, and provocation of ventricular fibrillation in an asymptomatic patient with Brugada syndrome. This report highlights the importance of investigating herbal medications in the detailed history of a patient in the cases of electrolyte disturbances and the potential role of hypokalemia for the induction of malignant dysrhythmias in Brugada syndrome. 


\section{References}

1 Brugada P: Brugada syndrome: an electrocardiographic diagnosis not to be missed Heart 2000;84:1-2.

-2 Araki T, Konno T, Itoh $\mathrm{H}$, Ino $\mathrm{H}$, Shimizu M Brugada syndrome with ventricular tachycardia and fibrillation related to hypokalemia. Circ J 2003;67:93-95.

3 Veltmann C, Borggrefe M, Schimpf R, Wolpert C: Images in cardiovascular medicine. Yew causes Brugada ECG. Circulation 2009; 119:1836-1837.

4 Rouleau F, Asfar P, Boulet S, Dube L, Dupuis JM, Alquier P, Vicor J: Transient ST segment elevation in right precordial leads induced by psychotropic drugs: relationship to the Brugada syndrome. J Cardiovasc Electrophysiol 2001;12:61-65.
Brugada P, Brugada J: Right bundle branch block, persistent ST segment elevation and sudden cardiac death: a distinct clinical and electrocardiographic syndrome. A multicenter report. J Am Coll Cardiol 1992;20: 1391-1396.

6 Antzelevitch C: The Brugada syndrome: diagnostic criteria and cellular mechanisms. Eur Heart J 2001;22:356-363.

7 Stewart PM, Wallace AM, Valentino R, Burt D, Shackleton CH, Edwards CR: Mineralocorticoid activity of liquorice: 11-betahydroxysteroid dehydrogenase deficiency comes of age. Lancet 1987;ii:821-824.

8 Sigurjonsdottir HA, Manhem K, Axelson M Wallerstedt S: Subjects with essential hypertension are more sensitive to the inhibition of 11 beta-HSD by liquorice. J Hum Hypertens 2003;17:125-131.
Krähenbühl S, Hasler F, Frey BM, Frey FJ, Brenneisen R, Krapf R: Kinetics and dynamics of orally administered 18 beta-glycyrrhetinic acid in humans. J Clin Endocrinol Metab 1994;78:581-585.

10 Sigurjonsdottir HA, Franzson L, Manhem K, Ragnarsson J, Sigurdsson G, Wallerstedt $S$ : Liquorice-induced rise in blood pressure: a linear dose response relationship. J Hum Hypertens 2001;15:549-552.

11 Clyburn EB, DiPette DJ: Hypertension induced by drugs and other substances. Semin Nephrol 1995;15:72-86.

12 Helfant RH: Hypokalemia and arrhythmias. Am J Med 1986;80:13-22.

13 Notarstefano P, Pratola C, Toselli T, Ferrari $\mathrm{R}$ : Atrial fibrillation and recurrent ventricular fibrillation during hypokalemia in Brugada syndrome. Pacing Clin Electrophysiol 2005;28:1350-1353. 